\title{
Spontaneous Esophageal Perforation Following Retropharyngeal Abscess Incision and Drainage
}

\author{
Theodore Klug ${ }^{1}$ and Courtney Shires ${ }^{1}$ \\ ${ }^{1}$ West Cancer Center
}

June 8, 2020

\begin{abstract}
Esophageal perforation is a rare but life-threatening condition1. Early detection and diagnosis are important to ensure good patient outcomes. The case that we present is of a patient that developed spontaneous esophageal perforation following irrigation and drainage of a retropharyngeal abscess.
\end{abstract}

Case Report: Spontaneous Esophageal Perforation Following Retropharyngeal Abscess Incision and Drainage Theodore Klug, MD, MPH ${ }^{1}$, Courtney B. Shires, MD, FACS ${ }^{1}$

${ }^{1}$ West Cancer Center, 7945 Wolf River Blvd, Germantown, TN 38104

Corresponding Author:

Theodore Klug, MD, MPH

West Cancer Center

7945 Wolf River Blvd, Germantown, TN 38104

901-491-1721

theodklu@gmail.com

Author Contributions

Courtney B. Shires, MD, FACS: Collected data, wrote and edited article

Theodore Klug, MD, MPH: Collected data, wrote and edited article

Financial Support: This particular research received no internal or external grant funding.

Conflicts of Interest: The authors report no relevant financial disclosures related to this current work.

Ethical Considerations: All issues related to ethics were taken into consideration throughout the study design and proposal and implemented during the research study itself. Informed consent was obtained, beneficence was made a top priority, and respect for confidentiality and privacy were upheld during the study and its various analysis and information assertation components.

\section{Abstract}

\section{Background}

Esophageal perforation is a rare but life-threatening condition ${ }^{1}$. Early detection and diagnosis are important to ensure good patient outcomes. At least half of esophageal perforations are iatrogenic, oftentimes caused 
by endoscopic instrumentation use in the upper gastrointestinal tract ${ }^{1}$. Still, about a third of esophageal perforations are spontaneous ${ }^{1}$. The scientific evidence that steers management of esophageal perforation is based primarily upon retrospective studies at single institutions, as well as on a few nationwide studies ${ }^{1-8}$. Randomized studies are virtually non-existent ${ }^{1}$. The case that we present is of a patient that developed spontaneous esophageal perforation following irrigation and drainage of a retropharyngeal abscess.

\section{Case Presentation}

A 49-year-old female, with a history of a retropharyngeal abscess status post irrigation and drainage on $5 / 3 / 20$, presented on $5 / 6 / 20$ to the emergency department in a large municipal city with increased pain at the surgical site. A computed tomography (CT) scan showed that the abscess had worsened, with it now extending into the posterior mediastinum from the aortic arch to the upper esophageal sphincter. The patient was taken to the operating room on 5/9/20 by Otolaryngology and Thoracic Surgery and underwent primary repair of cervical and thoracic esophageal perforation, sternocleidomastoid muscle flap reinforcement of the esophageal repair, and cervical and thoracic esophageal myotomy. Gastroenterology (GI) was also called into the operating room to assist with an esophagogastroduodenoscopy (EGD), which showed an esophageal tear $17 \mathrm{~cm}$ in length. The patient then had two esophageal stents placed, as well as a nasogastric tube and G-tube. The patient had an esophagram on 5/11/20, with no contrast extravasation. However, the patient did aspirate. The plan is to perform gastro-jejunal (GJ) conversion, per Interventional Radiology (IR), if no other complications ensue and the esophagus remains viable.

Her past medical history is significant for depression, schizophrenia, and nicotine abuse. Cultures of the abscess were taken, showing positivity for Prevotella bacteremia, and the patient was started on antibiotics. Otolaryngology, Thoracic Surgery, GI, and Medicine will continue to follow, with attention being paid to electrolyte replacement for a previous hyponatremia.

\section{Conclusions}

CT performed before initial incision and drainage of retropharyngeal abscess showed confinement to the retropharyngeal space (Figure 1). Following increased pain at the surgical site, repeat imaging showed extension into the mediastinum from the sagittal, axial, and coronal angles, respectively (Figures 2-6). Esophagram was performed and showed no extravasation, although the patient did aspirate (Figure 7). Based off of the patient history, we postulate that the esophageal perforation that occurred was a result of an increase in pressure due to patient activity. According to the patient, she had been yelling at her significant other for quite some time, with no other voice-related activity. With no episodes of vomiting or ingestion of a foreign body recorded prior to the esophageal rupture, combined with a chest x-ray showing no pneumoperitoneum, Boerrhave's syndrome was ruled out (Figure 8). Our case we believe is the first study showing a spontaneous esophageal perforation following an incision and drainage of a retropharyngeal abscess.

\section{Key Words}

Esophageal perforation; prevotella; retropharyngeal abscess; irrigation; drainage

\section{Key Clinical Message}

It is possible to have a spontaneous esophageal perforation following an incision and drainage of a retropharyngeal abscess. We believe our case is the first study showing such an event, which can become catastrophic if not addressed immediately.

\section{Case Presentation}

A 49-year-old female, with a history of a retropharyngeal abscess status post irrigation and drainage on $5 / 3 / 20$, presented on $5 / 6 / 20$ to the emergency department in a large municipal city with increased pain at the surgical site. A computed tomography (CT) scan showed that the abscess had worsened, with it now extending into the posterior mediastinum from the aortic arch to the upper esophageal sphincter. The patient was taken to the operating room on 5/9/20 by Otolaryngology and Thoracic Surgery and underwent primary 
repair of cervical and thoracic esophageal perforation, sternocleidomastoid muscle flap reinforcement of the esophageal repair, and cervical and thoracic esophageal myotomy. Gastroenterology (GI) was also called into the operating room to assist with an esophagogastroduodenoscopy (EGD), which showed an esophageal tear $17 \mathrm{~cm}$ in length. The patient then had two esophageal stents placed, as well as a nasogastric tube and G-tube. The patient had an esophagram on 5/11/20, with no contrast extravasation. However, the patient did aspirate. The plan is to perform gastro-jejunal (GJ) conversion, per Interventional Radiology (IR), if no other complications ensue and the esophagus remains viable.

Her past medical history is significant for depression, schizophrenia, and nicotine abuse. Cultures of the abscess were taken, showing positivity for Prevotella bacteremia, and the patient was started on antibiotics. Otolaryngology, Thoracic Surgery, GI, and Medicine will continue to follow, with attention being paid to electrolyte replacement for a previous hyponatremia.

\section{Discussion and Conclusions}

CT performed before initial incision and drainage of retropharyngeal abscess showed confinement to the retropharyngeal space (Figure 1). Following increased pain at the surgical site, repeat imaging showed extension into the mediastinum from the sagittal, axial, and coronal angles, respectively (Figures 2-6). Esophagram was performed and showed no extravasation, although the patient did aspirate (Figure 7). Based off of the patient history, we postulate that the esophageal perforation that occurred was a result of an increase in pressure due to patient activity. According to the patient, she had been yelling at her significant other for quite some time, with no other voice-related activity. With no episodes of vomiting or ingestion of a foreign body recorded prior to the esophageal rupture, combined with a chest x-ray showing no pneumoperitoneum, Boerrhave's syndrome was ruled out (Figure 8). Our case we believe is the first study showing a spontaneous esophageal perforation following an incision and drainage of a retropharyngeal abscess.

\section{List of Abbreviations}

computed tomography (CT); Gastroenterology (GI); gastro-jejunal (GJ); esophagogastroduodenoscopy (EGD)

\section{References}

1. Søreide JA, Viste A. Esophageal perforation: diagnostic work-up and clinical decision-making in the first 24 hours. Scand J Trauma Resusc Emerg Med . 2011;19:66. Published 2011 Oct 30. doi:10.1186/17577241-19-66

2. Sepesi B, Raymond DP, Peters JH. Esophageal perforation: surgical, endoscopic and medical management strategies. Curr Opin Gastroenterol. 2010;26:379-383. doi: 10.1097/MOG.0b013e32833ae2d7.

3. Ryom P, Ravn JB, Penninga L, Schmidt S, Iversen MG, Skov-Olsen P, Kehlet H. Aetiology, treatment and mortality after oesophageal perforation in Denmark. Dan Med Bull. 2011;58:A4267.

4. Vidarsdottir H, Blondal S, Alfredsson H, Geirsson A, Gudbjartsson T. Oesophageal perforations in Iceland: a whole population study on incidence, aetiology and surgical outcome. Thorac Cardiovasc Surg. 2010;58:476-480. doi:

5. Vallbohmer D, Holscher AH, Holscher M, Bludau M, Gutschow C, Stippel D, Bollschweiler E, Schroder W. Options in the management of esophageal perforation: analysis over a 12-year period. Dis Esophagus. 2010;23:185-190. doi: 10.1111/j.1442-2050.2009.01017.x.

6. Hermansson M, Johansson J, Gudbjartsson T, Hambreus G, Jonsson P, Lillo-Gil R, Smedh U, Zilling T. Esophageal perforation in South of Sweden: results of surgical treatment in 125 consecutive patients. BMC Surg. 2010;10:31. doi: 10.1186/1471-2482-10-31.

7. Udelnow A, Huber-Lang M, Juchems M, Trager K, Henne-Bruns D, Wurl P. How to treat esophageal perforations when determinants and predictors of mortality are considered. World J Surg. 2009;33:787796. doi: 10.1007/s00268-008-9857-5.

8. Bhatia P, Fortin D, Inculet RI, Malthaner RA. Current concepts in the management of esophageal perforations: a twenty-seven-year Canadian experience. Ann Thorac Surg. 2011;92:209-215. doi: 
10.1016/j.athoracsur.2011.03.131.
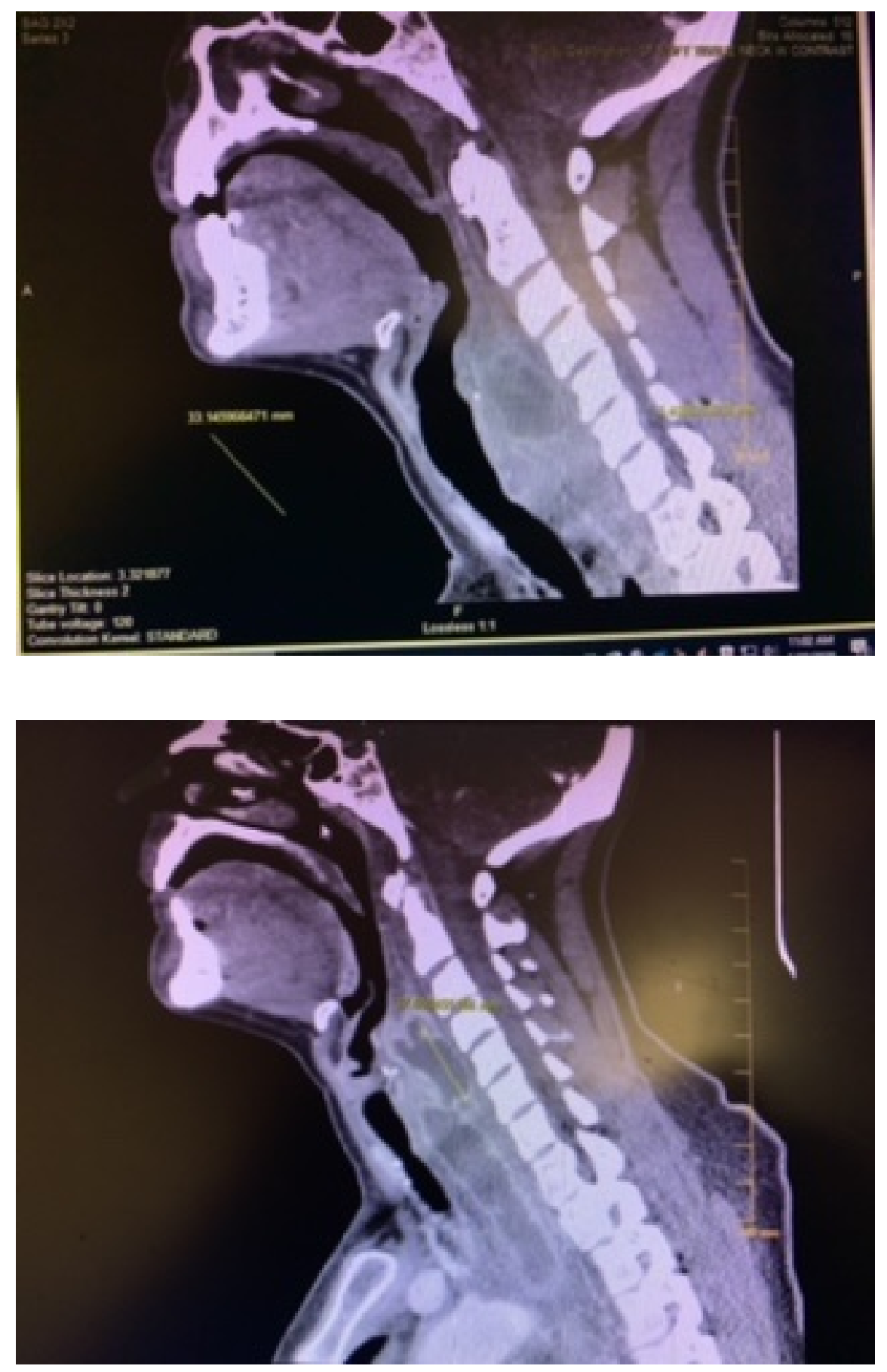

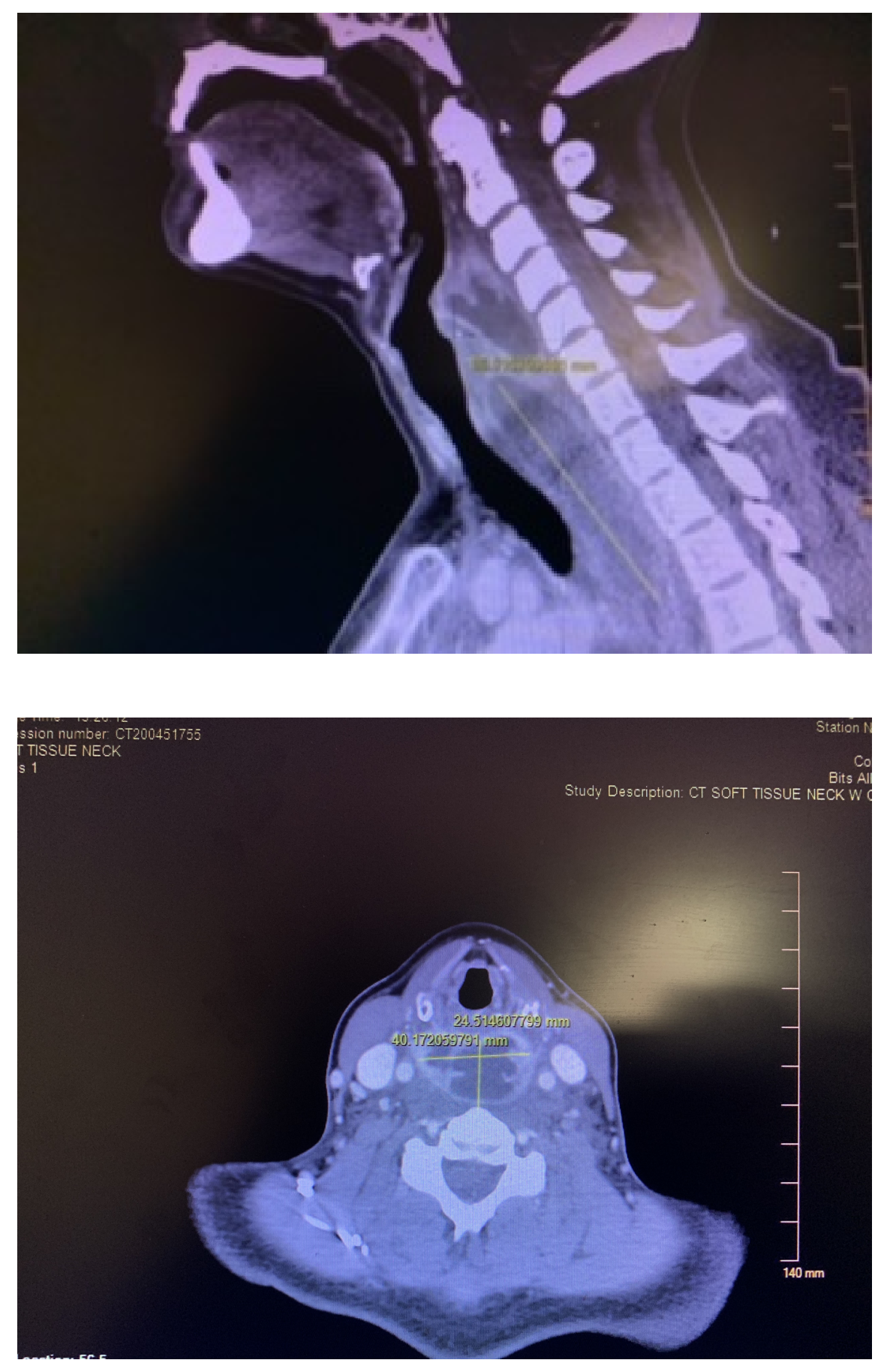

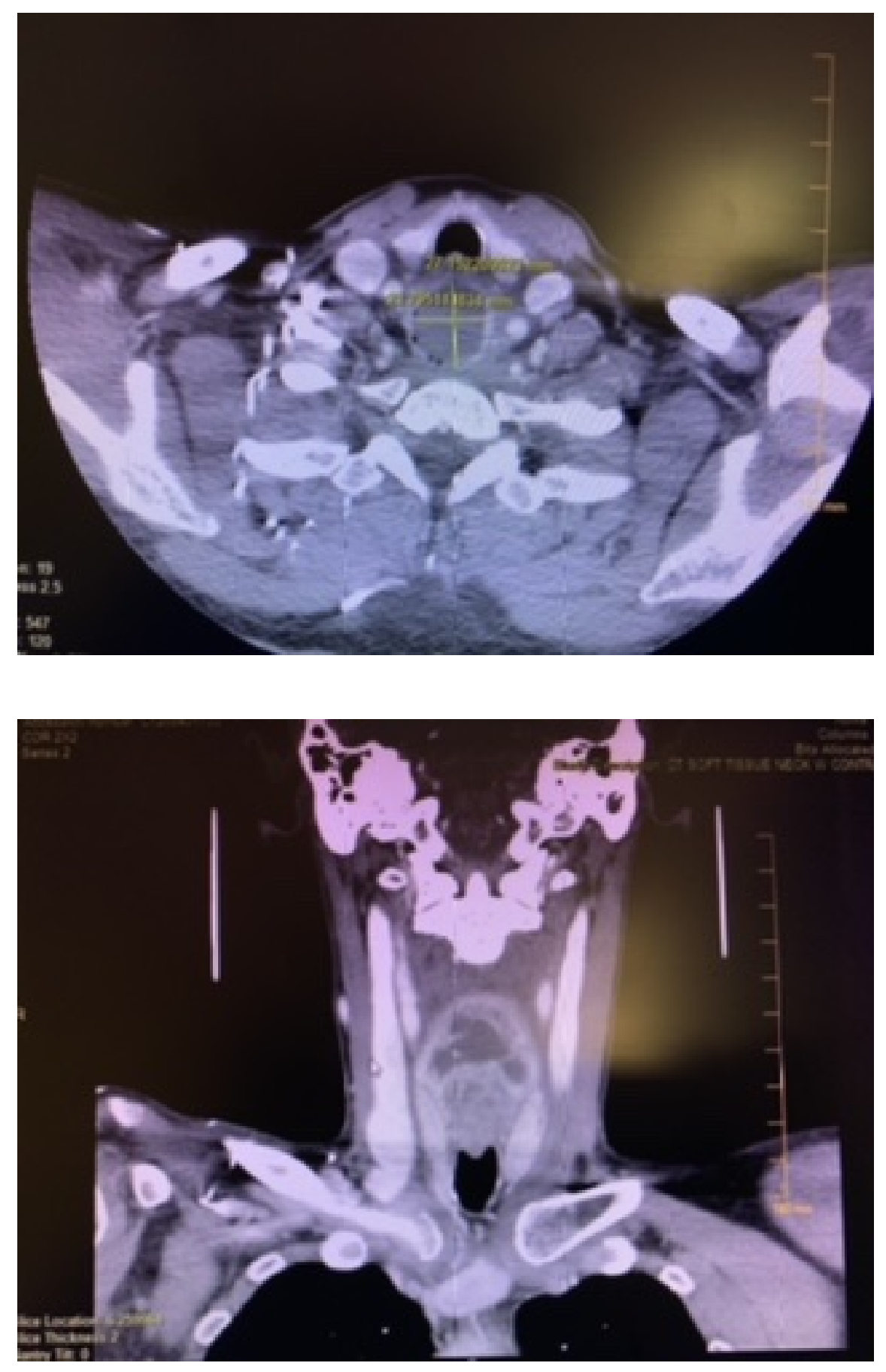


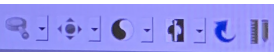
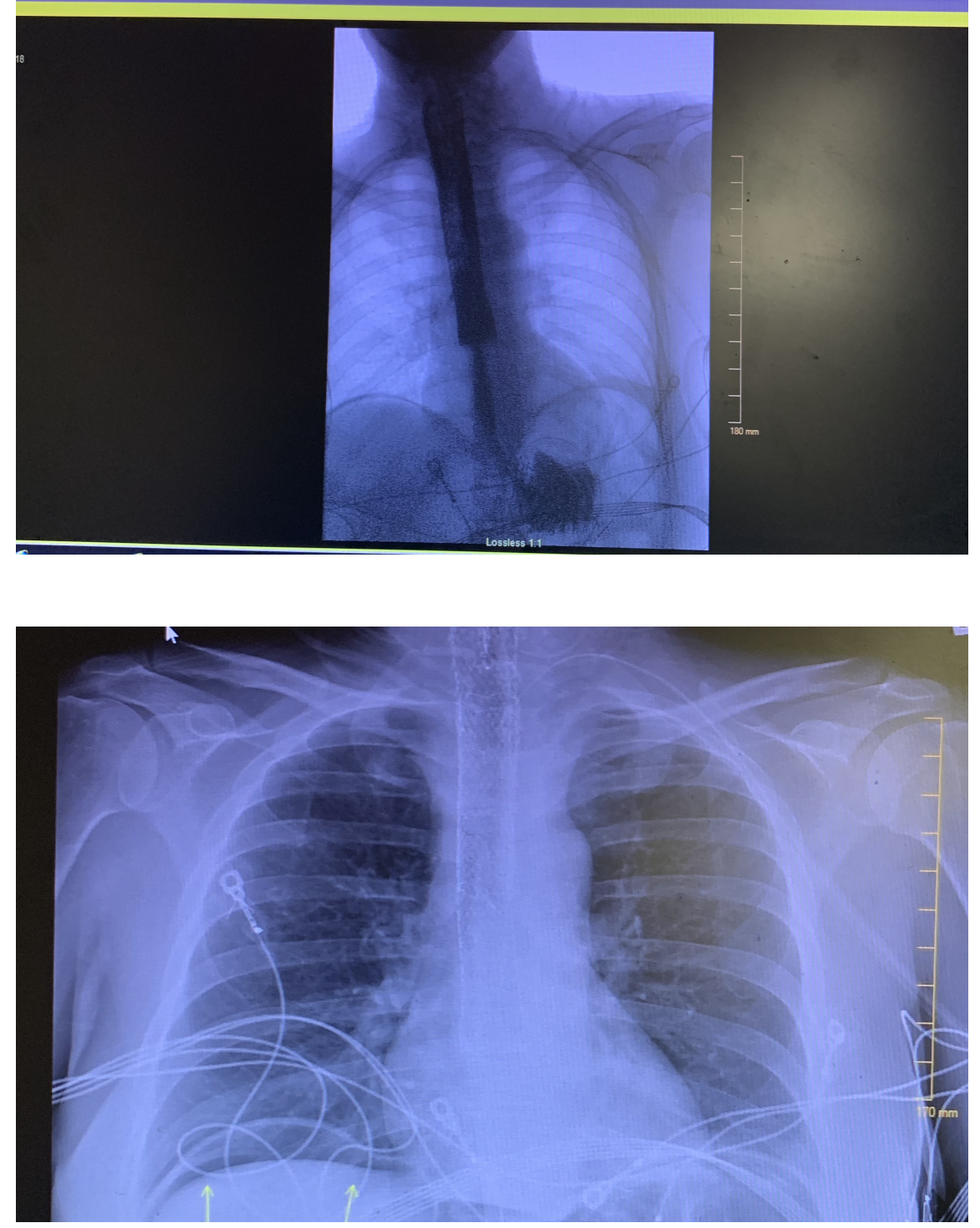